\title{
Resistance to Beta Lactam Antibiotics of Pseudomonas aeruginosa Isolated in Community Infections within HIV Infected Persons in Lomé-Togo
}

\author{
Majesté Ihou Wateba ${ }^{1}$, Kanyni Ekoue-Kouvahey², Abago Balaka³ \\ Komivi Tsatsu', Ousseni Tidjani ${ }^{1}$ \\ ${ }^{1}$ Department of Infectious Diseases and Pneumology, CHU Sylvanus Olympio, Lomé, Togo \\ ${ }^{2}$ Medical Center of NGO VISA, Lomé, Togo \\ ${ }^{3}$ Department of Internal Medicine, CHU Sylvanus Olympio, Lomé, Togo \\ Email: ${ }^{*}$ majeste7@yahoo.fr
}

Received 8 February 2014; revised 4 March 2014; accepted 11 March 2014

Copyright (C) 2014 by authors and Scientific Research Publishing Inc.

This work is licensed under the Creative Commons Attribution International License (CC BY). http://creativecommons.org/licenses/by/4.0/

\section{Open Access}

\begin{abstract}
Objective: Describe resistance to beta lactam antibiotics of Pseudomonas aeruginosa in community infection within HIV-1 infected persons. Methods: We have studied prospectively from June $15^{\text {th }}$ to December 31 2013 in the Clinic Hotel-Dieu and NGO VISA of Lomé, adult HIV-1 infected patients under anti retroviral therapy combining tenofovir, lamivudine and efavirenz for at least one year. The technique of agar diffusion susceptibility using discs of Ticarcillin + clavulanic acid is used to study the $\beta$-lactamase production. The diagnosis of species was performed by the chloroform test and the test for sensitivity to kanamycin and colistin. Results: Thirty five strains of Pseudomonas aeruginosa were obtained. The T-lymphocytes CD4 mediane was 575 cells $/ \mathrm{mm}^{3}$ of blood. Urine represented 15 cases, skin abscesses 11 cases, externa suppurate otitis 7 cases and vaginal swab for 2 cases. The phenotypes were: wild phenotypes 23 cases $(65.7 \%)$, resistant phenotypes 12 cases. Among resistant phenotypes, 4 were complex phenotype; 5 were ESBL phenotypes; 2 were hyper productive cephalosporinases phenotypes and 1 was a specific phenotype with impermeability to imipenem. Conclusion: The acquisition of resistance of Pseudomonas aeruginosa to beta lactam antibiotics in community infections among HIV-1 infected person incentives controls and promotes the rational use of antibiotics.
\end{abstract}

\section{Keywords}

HIV-1; Pseudomonas aeruginosa; Resistance Phenotypes; Beta Lactam Antibiotic

\footnotetext{
${ }^{*}$ Corresponding author.
}

How to cite this paper: Wateba, M.I., et al. (2014) Resistance to Beta Lactam Antibiotics of Pseudomonas aeruginosa Isolated in Community Infections within HIV Infected Persons in Lomé-Togo. World Journal of AIDS, 4, 81-84. 


\section{Introduction}

Pseudomonas spp. which is opportunistic pathogens often invades the tissue of their hosts and causes infection and bacteremia in immunocompromised persons (e.g. HIV/AIDS, cystic fibrosis, bronchiectasis and severe chronic obstructive pulmonary disease, burns, malignancy or diabetes mellitus...) [1] [2].

For many years, the $\beta$-lactam antibiotics have been widely associated with the treatment of Pseudomonas aeruginosa infections. However, resistance of $P$. aeruginosa to $\beta$-lactams can be acquired either by natural or inducible cephalosporinase produced by chromosomal origin, either by poor permeability of the antibiotic in the bacteria.

In Togo, many studies report levels of resistance of Pseudomonas aeruginosa to antibiotics, but none have specifically studied the problem in people infected with HIV-1 in community infection.

This study was then conducted in this population to find the production of $\beta$-lactamase in Pseudomonas aeruginosa using the disposition of the disk of Ticarcillin + clavulanate (TCC) and describe the resistance phenotypes of strains of Pseudomonas aeruginosa.

\section{Method}

The clinic Hotel Dieu and the medical center NGO VISA was the location for this study. This was a prospective study of all strains of Pseudomonas aeruginosa isolated in samples of patients infected with HIV-1 between June $15^{\text {th }}$, 2013 and December $31^{\text {st }}$, 2013. All samples are community infection and any patient had been hospitalized before.

The following antibiotic discs were used: Ticarcillin clavulanic acid, Ticarcillin, aztreonam, cefotaxime, ceftazidime, Cefsulodin, Imipenem.

The study focused on all Gram-negative bacillus, producing oxidase, identified as Pseudomonas aeruginosa.

The species diagnosis was made by the test of chloroform and with the test of sensitivity to kanamycin and colistin.

All patients are under antiretroviral therapy combined a single dose a day of tenofovir, lamivudine and efavirenz for at least one year.

The interpretation of susceptibility was based on the following characters:

The wild phenotype is:

1) The lack of synergy between the image of the disk Ticarcillin + clavulanic acid and those of the third generation cephalosporins (C3G) and/or aztreonam;

2) The presence of antagonism image between Imipenem and Cefsulodin;

3) The diameter of the inhibition zone around the Cefsulodin is substantially equal to the diameter of the inhibition around the aztreonam area.

The ESBL phenotype is:

1) The lack of synergy between the image of the disk Ticarcillin + clavulanic acid and those of the third generation cephalosporin (C3G) and/or aztreonam;

2) The contact around disks of Ticarcillin and those of C3G and aztreonam;

3) A reduction of sensitivity diameter around ceftazidime (diameter $\leq 22 \mathrm{~mm}$ ) and/or aztreonam (diameter $\leq$ $27 \mathrm{~mm}$ ).

The hyperproductive cephalosporinase phenotype:

In the case of partial derepression we have:

1) A lack of synergy between the image of the disc ticarcillin + clavulanic acid and those of C3G and/or aztreonam;

2) A presence of an antagonism image between Imipenem and Cefsulodin;

3) The diameter of the inhibition zone around the ceftazidime is smaller than the diameter of the inhibition zone around the aztreonam;

4) The largest of the diameter of the inhibition zone of the disk of Cefsulodin defined the type is resistant or intermediate.

The specific phenotype of the impermeability to Imipenem corresponds:

1) In the absence of synergy between the image disc ticarcillin + clavulanic acid and those of C3G and/or aztreonam;

2) The presence of antagonism image between Imipenem and Cefsulodin; 
3) The diameter of the inhibition zone around the Cefsulodin is substantially equal to the diameter of the inhibition zone around the aztreonam;

The result is the resistance of Imipenem.

The complex phenotype: the interpretation of the complex phenotype is difficult.

The immune status appreciated by CD4 count, the sex, the nature of the biological product, the resistance phenotype of the Pseudomonas aeruginosa were the parameters we had studied.

\section{Results}

\subsection{Epidemiological Data}

Samples representative of 18 men and 17 women were collected. The mean age was $32 \pm 6$ years old.

\subsection{Origin of the Strains}

Thirty five strains of $P$. aeruginosa were isolated. Sixteen came from NGO VISA and 19 from medical clinic Hôtel Dieu (Table 1).

\subsection{Biological Data}

All patients were infected with HIV-1 and the median T-lymphocyte CD4 count was 575 cells per $\mathrm{mm}^{3}$ of blood with a range of 495 and 755 cells $/ \mathrm{mm}^{3}$. Four pathological products were analyzed. It was urine for 15 cases, 11 cases for skin abscesses, externa suppurate otitis in 7 cases and 2 cases of vaginal swab.

\subsection{Phenotypes}

Twenty three strains of Pseudomonas aeruginosa was wild-type Twelve isolates were resistant. Among them, the complex phenotypes has represented 4 cases, the ESBL phenotypes 5 cases, the hyper productive Cephalosporinases 2 cases and 1 case of Specific impermeability to imipenem (Table 2).

\section{Discussion}

All strains which were isolated were from community infection. Among them, 12 had presented resistance phenotype. Among resistant strains, 4 were complex phenotypes and 2 come from skin abscess, 5 were ESBL phenotypes, 2 were cephalosporinases hyper productive phenotypes and only 1 case of specific impermeability phenotype to imipenem coming from a vaginal swab. This is alarming because conventionally, resistant strains are

Table 1. Distribution of Pseudomonas aeruginosa strains isolated by service.

\begin{tabular}{cc} 
Services & Number \\
\hline Hotel Dieu Medical Clinic & 19 \\
NGO VISA & 16 \\
Total & 35 \\
\hline
\end{tabular}

Table 2. Distribution of phenotypes of Pseudomonas aeruginosa strains isolated by the technique of Mueller Hinton agar.

\begin{tabular}{cc}
\hline Phenotypes isolated & Number (proportion) \\
Wild phenotypes & $23(65.7 \%)$ \\
Resistant phenotype & $12(34.3 \%)$ \\
Complex phenotypes & 4 \\
ESBL phenotypes & 5 \\
Hyperproduitive Cephalosporinases & 2 \\
Specific impermeability to imipenem & 1 \\
\hline
\end{tabular}


nosocomial. The reason can be the uncontrolled use of antibiotics by self-medication and the use of biocides which can be found easily on the sidewalks belly markets in most developing countries including Togo [3] [4]. In addition, there is a flooding of the market of developing countries by antibiotics, from the newly industrialized countries of Africa and Asia, which are not always WHO prequalified. This casts doubt on their true quality. But it is clear they are widely prescribed because they are more affordable for consumers. Very often, people in poor countries go to the hospital after a failed of the first self administered therapy which is often made by a cocktail of antibiotics, analgesics and anti-inflammatory [3]. Moreover, the pathogenicity of Pseudomonas infections is increased in the cause of immunodeficiency [5] [6]. However, in our study, all patients, although infected with HIV-1, was not immunocompromised (T-CD4 count of all patients were more than $575 \mathrm{cells} / \mathrm{mm}^{3}$ of blood) and all of them is under antiretroviral therapy for at least one year. Other authors have found high proportions of Pseudomonas aeruginosa resistant to beta lactamins in patients not infected with HIV in both community and hospital settings [7]. This phenomenon invariably affect everybody where the need of the proper use of antibiotics. In addition, the emergence of a resistant strain to imipenem which is supposed to represent a real alternative in the treatment of multi-resistant bacteria to beta lactam [8] is worrisome. So there is a real danger because it is clear that today, no antibiotic can escape the phenomenon of bacterial resistance, even in community-acquired infections. Then the establishment of a monitoring system and the strict application of hygiene and standard of good prescription of antibiotics will be helpful.

\section{Conclusion}

At the end of our study, 35 strains of Pseudomonas aeruginosa were isolated from different products in ambulatory HIV-1 infected patients. Although they are HIV-1 infected, the resistant phenotype observed didn't show a particular difference compared to HIV uninfected persons. But we remark that $P$. aeruginosa is becoming more and more resistant to beta lactamin even isolated in community infection. Therefore a rigorous surveillance is necessary in the hope to control community strains and to improve a better prescription of antibiotic in the community.

\section{Conflict of Interest}

We have no conflict of interest to declare. This work is a personal work of the authors.

\section{References}

[1] Liu, P.V. and Mercer, C.B. (1963) Growth, and Virulence of Toxigenicity Pseudomonas Aeruginosa. The Journal of Hygiene, 61, 485-491. http://dx.doi.org/10.1017/S0022172400021100

[2] Feldman, M., Bryan, R., Rajan, S., Scheffler, L., Brunnert, S., Tang, H. and Prince, A. (1998) Role of Flagella in Pathogenesis of Pseudomonas aeruginosa Pulmonary Infection. Infection and Immunity, 66, 43-51.

[3] Bignandi, A. (2005) Vente illicite du médicament. Thèse de Pharmacie, Lomé, 34-39.

[4] MS/DGS/DES (2006) Répertoire des établissements privés de santé au Togo. 23-25.

[5] Jeannat, K. and Plesiat, P. (2005) Therapeutic Implication of Antibiotic Resistance in Pseudomonas. Lettre de l'infectiologue, 7.

[6] Navon-Venezia, S., Ben Ami, R. and Carmeli, Y. (2005) Update on Pseudomonas aeruginosa and Acinetobacter baumanii Infections in the Healthcare Setting. Current Opinion in Infectious Diseases, 18, 306-313. http://dx.doi.org/10.1097/01.qco.0000171920.44809.f0

[7] Faure, K., Kipnis, E. and Gueri, B. (2008) Prise en charge des pneumonies à Pseudomonas aeruginosa. Rev Tun Infectiol., 2, 1-8.

[8] Ait El Kadi, M., Aghrouch, M., Seffar, M., Elharti, J., Bouklouze, A., Cherrah, Y., et al. (2006) Prévalence des souches d'Acinetobacter baumannii et de Pseudomonas aeruginosa résistantes à l'imipénème par production de métallo- $\beta$ lactamases. Med Mal Infect., 36, 386-389. http://dx.doi.org/10.1016/j.medmal.2006.05.003 